\title{
Intake of Medusahead by Sheep: Influence of Supplements, Silica and Individual Animal Variation
}

\author{
Juan J. Villalba and Elizabeth A. Burritt*
}

\begin{abstract}
Grazing represents a sustainable alternative for the control of medusahead infestations but intake of medusahead by ruminants is typically low. We determined in sheep whether (1) high-energy supplements enhance medusahead intake and preference relative to a treatment without supplementation and (2) individual differences in medusahead intake transfer to individual differences in the ingestion of a silica-containing ration. Groups of lambs $(n=10)$ were individually penned and randomly assigned to three supplementation treatments: (1) Control (no supplement); (2) Calcium (Ca) Propionate (beet pulp:barley: Ca propionate, 67:30:3); or (3) Yeast (beet pulp: barley: yeast culture, $65: 30: 5$ ). After supplementation, all animals had ad libitum access to medusahead in late vegetative to mid-reproductive (Trial 1), and late-reproductive phenological stages (Trial 2). Medusahead preferences were assessed by offering sheep a choice between medusahead and tall fescue hay. Plant part preferences were assessed by offering a choice between medusahead tops (mostly seedheads and awns) and mid-plant parts (mostly stems + leaves). Intake of medusahead was low and cyclic, declining towards the end of each trial $(\mathrm{P}<0.0001)$ and there were no treatment differences $(\mathrm{P}>0.10)$. Lambs preferred tall fescue hay to medusahead and medusahead tops to mid-plant parts $(\mathrm{P}<0.0001)$. Supplemented lambs gained more weight than control lambs $(\mathrm{P}<0.10)$. Thus, supplemented lambs performed better than nonsupplemented controls without reducing their intake of medusahead. In Trial 3, two new groups of lambs were formed based on their intake of medusahead during Trials 1 and $2(n=10)$. One group consistently ate more medusahead and more of a ration containing silica (alfalfa: silica, 97:3) than the other $(\mathrm{P}<0.10)$. A significant and consistent degree of individual variation was measured among lambs-irrespective of treatment—regarding their ability to ingest medusahead and silica. This variation represents a promising option for maximizing use of medusahead by livestock in rangelands.
\end{abstract}

Nomenclature: Medusahead, Taeniatherum caput-medusae (L.) Nevski.

Key words: Invasive grasses, invasive plants, foraging behavior, supplement, targeted grazing.

Medusahead (Taeniatherum caput-medusae (L.) Nevski) is an annual grass native to the Mediterranean region, which has invaded millions of acres in the Pacific Northwest, California, Utah and Nevada (Zimmerman et al. 2002). The extent and severity of the problem make efforts for its control an essential endeavor for the profitability and sustainability of these regions. Chemical control of the weed can be effective but it has potential negative side effects on soils and native plants (Davies and Johnson 2008), it may not be a reliable method of control from year to year, and it is expensive, which many ranchers

\footnotetext{
DOI: $10.1614 /$ IPSM-D-14-00072.1

* Associate Professor and Extension Assistant Professor, Department of Wildland Resources, Utah State University, Logan, Utah, 84322. Corresponding author’s E-mail: juan.villalba@usu.edu
}

cannot afford. In addition, medusahead can grow in rough terrain across rangelands, making difficult and impractical the application of chemical control methods.

Grazing represents a sustainable, efficient, and low-cost alternative to chemical control. Instead of destroying the plant with costly chemicals, grazers could use the carbohydrates, protein and minerals in the plant for their own nutrition while reducing its abundance and competitive ability in the plant community. Nevertheless, intake of medusahead by ruminants is typically low, particularly as the plant matures during the growing season (Davy et al. 2009; Doran 2008). It has been claimed that livestock reject medusahead during grazing due to its low nutritive value (Bovey et al. 1961; Murphy and Turner 1959) and special attention has been given to the antinutritional effects of the high silica content of the weed (Swenson et al. 1964). In fact, it has been claimed that high silica 


\section{Management Implications}

High-energy supplements containing glucogenic agents (Ca propionate) or yeast cultures (S. cerevisiae fermentation products) did not increase medusahead use by lambs relative to unsupplemented animals. However, our results suggest that supplementation programs can improve the production of grazing animals in targeted grazing treatments without depressing the ingestion of medusahead relative to unsupplemented (i.e., forced to ingest medusahead) animals. Intake of medusahead was low and cyclic suggesting a metabolic constraint which restricts the amount of forage consumed by an animal on a daily basis. However, a significant degree of individual variation was measured in the animals under study-irrespective of treatment-suggesting that these differences were more relevant than the treatments used to enhance intake of the weed. Individual differences in medusahead intake by livestock represents a promising option for maximizing use of medusahead by livestock on rangelands.

concentration represents a plant defense mechanism against herbivory (McNaughton et al. 1985).

Given the low use of medusahead by grazing herbivores, attempts have been made at enticing livestock to consume the weed through the use of attractants (Bovey et al. 1961). However, liquid molasses or salt applied directly on medusahead during the late, dry season did not affect use by cattle or sheep (Davy et al. 2009; Doran 2008). In contrast, recent research in our lab suggests high-energy supplements increase use of medusahead by sheep compared to sheep not supplemented (Hamilton et al. 2014; Montes-Sanchez and Villalba, unpublished results). Nevertheless, the increase was modest and substantially below the intake capacity of the animals studied. Dietary ingredients such as Ca propionate and yeast cultures (e.g., Saccharomyces cerevisiae fermentation products) may enhance medusahead intake and animal performance relative to the high-energy supplements previously studied. Propionate supplementation may improve ruminant nutrition when glucogenic precursors are inadequate due to low-quality diets and/or increased energy demands (Mulliniks et al. 2011). Yeast cultures, on the other hand, increase cellulose degradation by rumen microbes (Williams et al. 1991) and may increase medusahead use by grazing animals.

Finally, it is clear from previous studies (Hamilton et al. 2014; Montes-Sanchez and Villalba, unpublished results) that some individuals have a greater capacity to consume medusahead than others-irrespective of the supplementation program-suggesting that individual variation is an important component underlying the ability of livestock to consume medusahead. Thus, the objectives of this study were to determine in sheep whether: (1) ingredients such as $\mathrm{Ca}$ propionate and yeast culture mixed in high-energy supplements enhance medusahead intake and preference relative to a treatment without supplementation, and
(2) individual differences in medusahead intake influence the ingestion of a silica-containing ration.

\section{Materials and Methods}

The study was conducted at the Green Canyon Ecology Center in Logan, UT according to procedures approved by the Utah State University Institutional Animal Care and Use Committee (Approval \# 1551). Upon arrival, all animals were dewormed for internal and external parasites (Ivermectin; $0.2 \mathrm{mg} \mathrm{kg}^{-1}$ [3.18 $10^{-6}$ ounces $\left.\mathrm{lb}^{-1}\right]$ of $\mathrm{BW}$ ) and vaccinated against clostridial diseases (Clostridium perfringens types C \& D and tetanus toxoid; $2 \mathrm{ml}[0.07$ ounces] lamb ${ }^{-1}$ ).

All lambs were individually penned outdoors, under a protective roof in individual, adjacent pens measuring 1.5 by $2.5 \mathrm{~m}$. Throughout the study, lambs had free access to water and trace mineral salt blocks.

Medusahead and Fescue Hay. Naturally established stands of medusahead (Taeniatherum caput-medusae (L.) Nevski) growing on private land within the city limits of Paradise, Cache County, Utah $\left(41^{\circ} 34^{\prime} 03^{\prime \prime} \mathrm{N} 111^{\circ} 50^{\prime} 02^{\prime \prime} \mathrm{W}\right)$ were used in the study. Medusahead was harvested using a lawnmower (Husqvarna HD800BBC with a bag catcher) in the late vegetative -early reproductive stage (from emergence of awns to early emergence of seedhead) during feeding Trial 1 (June 3 to June 13, 2013), and during the late reproductive stage (mature) for feeding Trial 2 (June 24 to June 30, 2013). Medusahead was harvested fresh daily, transported to the Green Canyon Ecology Center, and fed to lambs upon arrival. The particle size of the harvested material was $5 \mathrm{~cm}$ (1.97 inches) in length. Given this size, awns from the mature madusahead plants were not chopped or broken during the harvesting process.

Endophyte-free tall fescue (E-; Lolium arundinaceum [Schreb.] Darbysh) hay was harvested and baled in spring of 2013. The experimental hay was passed through a hydraulic bale grinder (Gehl commercial forage grinder) and cut into lengths of 1 to $4 \mathrm{~mm}$ (0.04 to 0.16 inches) particle size. The ground material was bagged in $20 \mathrm{~kg}$ moisture resistant bags and stored in a shaded building.

Animals and Pre-Conditioning Period. Thirty commercial crossbred lambs (3 mo of age) with an average initial body weight (BW) of $28 \pm 1.3 \mathrm{~kg}$ were blocked by BW and randomly assigned to one of three treatment groups $\left(10\right.$ lambs group $\left.^{-1}\right)$. Treatment groups were: (1) Control (lambs received no supplement), or a high energy/protein ratio supplement ( 1 to $2 \mathrm{~mm}$ particle size) containing either (2) Calcium Propionate (67\% beet pulp, 30\% barley, 3\% Ca propionate; Sigma-Aldrich, St. Louis, MO, USA), or (3) Yeast (65\% beet pulp, $30 \%$ barley, $5 \%$ yeast culture: Saccharomyces cerevisiae fermentation product; Diamond V XP, Diamond V Mills, Cedar Rapids, IA) 
Table 1. Nutritional composition of medusahead, supplements, endophyte-free tall fescue hay, and silica diet during the study.

\begin{tabular}{|c|c|c|c|c|}
\hline \multirow[b]{2}{*}{ Item } & \multicolumn{4}{|c|}{$\% \mathrm{DM}$} \\
\hline & $\mathrm{CP}^{\mathrm{g}}$ & $\mathrm{NDF}^{\mathbf{h}}$ & $\mathrm{ADF}^{\mathrm{i}}$ & $\operatorname{AIA}^{\mathrm{j}}$ \\
\hline \multicolumn{5}{|l|}{ Medusahead } \\
\hline Trial $1^{\mathrm{a}}$ & $10.8 \pm 0.3$ & $62.0 \pm 1.2$ & $46.3 \pm 1.5$ & $16.0 \pm 2.1$ \\
\hline Trial $1^{b}$ & $10.2 \pm 0.2$ & $60.4 \pm 1.3$ & $40.1 \pm 1.2$ & $10.7 \pm 1.1$ \\
\hline Trial $2^{c}$ & $8.5 \pm 0.2$ & $58.2 \pm 0.6$ & $37.8 \pm 0.5$ & $8.1 \pm 0.5$ \\
\hline Tops of the plant & $10.3 \pm 0.3$ & $62.2 \pm 1.4$ & $38.8 \pm 1.6$ & $8.2 \pm 0.5$ \\
\hline Mid-parts of the plant & $7.5 \pm 0.2$ & $62.7 \pm 1.3$ & $42.6 \pm 1.2$ & $9.9 \pm 1.4$ \\
\hline \multicolumn{5}{|l|}{ Supplements } \\
\hline Ca Propionate ${ }^{\mathrm{d}}$ & $10.3 \pm 0.4$ & $31.0 \pm 1.7$ & $19.3 \pm 1.6$ & $\mathrm{ND}^{\mathrm{k}}$ \\
\hline Yeast $^{\mathrm{e}^{\mathrm{T}}}$ & $10.1 \pm 0.3$ & $30.0 \pm 1.2$ & $18.5 \pm 1.4$ & ND \\
\hline Tall fescue hay & $6.7 \pm 0.2$ & $62.1 \pm 1.4$ & $36.7 \pm 0.4$ & $8.5 \pm 1.2$ \\
\hline Alfalfa : silica diet ${ }^{f}$ & $15.1 \pm 0.6$ & $39.8 \pm 0.8$ & $29.2 \pm 0.4$ & ND \\
\hline \multicolumn{5}{|c|}{${ }^{2}$ Late vegetative/early reproductive stage: June 3 to June 7 . } \\
\hline \multicolumn{5}{|c|}{${ }^{\mathrm{b}}$ Early/mid-reproductive stage: June 8 to June 13 . } \\
\hline \multicolumn{5}{|c|}{${ }^{\circ}$ Late reproductive stage: June 24 to June 29 period. } \\
\hline \multicolumn{5}{|c|}{${ }^{\mathrm{d}}$ High-energy supplement: $67 \%$ beet pulp, $30 \%$ barley, $3 \%$ Ca propionate. } \\
\hline \multirow{2}{*}{\multicolumn{5}{|c|}{$\begin{array}{l}{ }^{\mathrm{e}} \text { High-energy supplement: } 65 \% \text { beet pulp, } 30 \% \text { barley, } 5 \% \text { yeast culture. } \\
{ }^{\mathrm{f}} 97 \% \text { alfalfa, } 3 \% \mathrm{SiO} 2 .\end{array}$}} \\
\hline & & & & \\
\hline \multicolumn{5}{|l|}{${ }^{\mathrm{g}}$ Crude protein. } \\
\hline \multicolumn{5}{|l|}{${ }^{\mathrm{h}}$ Neutral detergent fiber. } \\
\hline \multicolumn{5}{|l|}{${ }^{\mathrm{i}}$ Acid detergent fiber. } \\
\hline \multicolumn{5}{|l|}{ ¡Acid insoluble ash. } \\
\hline${ }^{\mathrm{k}}$ Not determined. & & & & \\
\hline
\end{tabular}

(Table 1). Lambs were familiarized to the experimental protocol for $11 \mathrm{~d}$ before the onset of Trial 1. Lambs in Groups 2 and 3 were fed $500 \mathrm{~g}$ (1.1 lbs) of their respective supplements at 8:00 A.M. and refusals were collected at 8:20 A.M. Subsequently, all lambs were offered alfalfa pellets until 2:00 P.M., when refusals were collected. Lastly, each lamb received $500 \mathrm{~g}$ of tall fescue hay until 4:00 P.M. No other food was offered until the next day.

Trial 1-Medusahead in Late Vegetative/Early Reproductive Stage. The objectives of this trial were to determine in sheep (1) whether high-energy supplements (Calcium Propionate or Yeast Culture) enhance medusahead intake and preference relative to a treatment without supplementation and (2) preference for different plant parts when medusahead is at the late vegetative/early reproductive stage of growth.

Medusahead Intake. Each day from 8:30 A.M. to 8:50 A.M. lambs in Groups 2 and 3 were offered their respective supplements in ad libitum amounts. Supplements were then removed and intake was calculated by subtracting the amount refused from the amount offered. All animals then received freshly harvested and chopped medusahead in ad libitum amounts in their wooden feeders from 9:00 A.M. to 2:00 P.M. Throughout the trial lambs were offered between 200 and $500 \mathrm{~g}$ of medusahead (as-fed basis) depending on the ability of each individual lamb to consume medusahead. Feeders were monitored every hour and additional amounts of medusahead were added to the feeders when the amounts remaining were below $20 \%$ of the amounts offered initially.

Following removal of medusahead, all groups received ad libitum amounts of endophyte-free tall fescue hay from 2:00 P.M. to 4:00 P.M. Tall fescue intake for each animal was estimated at 4:00 P.M. and no other food was offered until the next day at 8:30 A.M. The trial was conducted from June 3 to June 12, 2013.

Choice Test-Medusahead vs. Tall Fescue Hay. On June 13, after collecting supplement refusals, and from 9:00 A.M. to 2:00 P.M. all lambs received a choice of tall fescue hay and medusahead. After the choice, tall fescue hay and medusahead refusals were collected and intake was estimated as described before. No other food was offered until the following day. On June 14, all animals were weighed to estimate average daily gains (ADG) and then offered alfalfa pellets in ad libitum amounts until June 16. 
Choice between Plant Parts. On June 16 alfalfa pellets were removed at 4:00 P.M. from all feeders and no food was offered until the following day. On June 17, the tops of medusahead platns (estimated by observation 70 to $80 \%$ seedheads and awns) were collected by adjusting the lawnmower to its highest setting in order to cut the weed to a height of 9 to $10 \mathrm{~cm}$. Then, the lawnmower was adjusted to its lowest setting ( 3 to $5 \mathrm{~cm}$ ) and the same areas harvested before were mowed to collect the mid parts of the plant (estimated by observation 70 to $80 \%$ stems and leaves). From 9:00 A.M. to 2:00 P.M. all lambs received a choice of medusahead collected at the two plant heights (tops vs. mid parts). After the choice, medusahead refusals were collected at 4:00 P.M. and intake of each plant part was estimated. All animals were then offered alfalfa pellets in ad libitum amounts until July 8.

Trial 2-Medusahead in Late Reproductive Stage. The objective of this trial was to determine in sheep whether high-energy supplements (Calcium Propionate or Yeast Culture) enhance medusahead intake and preference relative to a treatment without supplementation when medusahead is at the late reproductive stage of growth.

Lambs were fed their respective supplements and medusahead as described in "Medusahead Intake" for Trial 1 (see above). However, no tall fescue hay or any other food was offered after medusahead refusals were collected at 2:00 P.M. Medusahead (late reproductive stage) was offered from June 24 to June 29, 2013. A choice test between medusahead and tall fescue hay was conducted on June 30, 2013 as described in "Choice Test-Medusahead vs. Tall Fescue Hay" for Trial 1 (see above).

Trial 3-Individual Variation and Intake of Silica. Throughout Trials 1 and 2 results indicated that some individuals consistently ate more medusahead than others, regardless of supplemental treatment. Thus, two new groups $(n=10)$ of lambs were created based on their consumption of medusahead: (1) Eaters, and (2) nonEaters. The criteria for creating these 2 new groups of lambs was that animals had to consume an average $\geq 6.5 \mathrm{~g} \mathrm{~kg}^{-0.75}$ (Eaters) or between 0 and $5 \mathrm{~g} \mathrm{~kg}^{-0.75}$ (nonEaters) of medusahead during Trials 1 and 2. Lambs from the Control $(n=3$ and 4$)$, Yeast $(n=3$ and 4$)$, and Ca Propionate ( $n=4$ and 2) groups formed the Eaters and nonEaters groups, respectively.

Each day at 8:30 A.M. lambs in both groups received a ration of ground alfalfa pellets containing $3 \%$ silica in ad libitum amounts. At 2:00 P.M. refusals were collected and weighed. Following removal of feeds, all groups received ad libitum amounts of ground endophyte-free tall fescue hay (1 to $2 \mathrm{~mm}$ particle size) from 2:00 P.M. to 4:00 P.M. Tall fescue intake for each animal was recorded as described before and no other food was offered until the next day at
8:30 A.M. The trial was conducted for four consecutive days (July 9 to July 12, 2013). Subsequently, both groups were offered alfalfa pellets in ad libitum amounts until July 21, when refusals were collected at 4:00 P.M. On July 22, lambs in both groups were fed ground alfalfa pellets (without silica) from 8:30 A.M. to 2:00 P.M., and then ground endophyte-free tall fescue hay from 2:00 P.M. to 4:00 P.M. as described before. Alfalfa was fed for four consecutive days (July 22 to July 25, 2013) in order to establish baseline intake of ground alfalfa by lambs when silica was absent from the diet.

Chemical Analyses. Every day that medusahead was fed, representative samples of the weed were placed in plastic bags and transported to a freezer where they were kept at $-20 \mathrm{C}(-4 \mathrm{~F})$ and subsequently freeze dried. Dried medusahead, tall fescue hay, supplement and silica-alfalfa samples were ground through a Wiley mill with a $1-\mathrm{mm}$ screen, and analyzed for dry matter (Method 930.15 AOAC 2000), neutral detergent fiber (NDF), acid detergent fiber (ADF) (Van Soest et al. 1991), and nitrogen (N) (Method 990.03 AOAC 2000). Medusahead and tall fescue hay were also analyzed for acid insoluble ash (Van Keulen and Young 1977) as an estimate of silica content.

Representative samples of the offered and refused feeds were placed in paper bags and dried in a forced-air oven at $60 \mathrm{C}$ for $48 \mathrm{~h}$ to estimate dry matter content, in order to express intake values on a dry matter basis.

Statistical Analyses. Analyses were computed using a mixed model (MIXED procedure; SAS Inst., Inc. Cary, NC; Version 9.1 for Windows). The variance-covariance structure used were those (autoregressive order-1, compound symmetry, variance components), which yielded the lowest Bayesian information criterion. The model diagnostics included testing for a normal distribution of the error residuals and homogeneity of variance. Means were analyzed using pairwise differences (DIFF) of least squares means (LSMEANS).

Trials 1 and 2. Food intake, expressed as grams of feed consumed (dry matter basis) $\mathrm{kg}^{-1}$ of metabolic body weight $\left(\mathrm{BW}^{0.75}\right)$, was analyzed as a mixed model with repeated measures (day) and lambs (random factor) nested within treatment group (fixed factor).

During preference tests, food intake and preference ([intake of a plant species/total intake] $\times 100)$ were analyzed as a mixed model with lambs (random factor) nested within treatment group (between-subject factor). Plant species (medusahead, tall fescue hay) or part (seedheads with awns, stems + leaves) were the withinanimal factor in the analyses.

ADG were analyzed as a mixed model with lambs (random factor) nested within group (fixed factor). 
Trial 3. Medusahead and alfalfa-silica intake, expressed as grams of feed consumed (dry matter basis) $\mathrm{kg}^{-1}$ of metabolic body weight $\left(\mathrm{BW}^{0.75}\right)$, was analyzed as a mixed model with repeated measures (d) and lambs (random factor) nested within group (Eaters, nonEaters). Baseline intake of ground alfalfa by lambs (i.e., when silica was absent) was a covariate in the analysis.

\section{Results}

Nutritional Analyses. Nutritional analyses for the feeds used in the study are reported in Table 1. Medusahead crude protein $(\mathrm{CP})$ content declined with maturity, although acid insoluble ash (AIA) and acid detergent fiber $(\mathrm{ADF})$ concentrations were greater in less mature medusahead plants (e.g., during Trial 1). The fraction containing mostly medusahead awns (top parts) had greater concentrations of $\mathrm{CP}$ and lower concentrations of ADF than the fraction containing mostly stems + leaves (mid parts). Tall fescue hay had lower concentration of CP (Trials 1 and 2), ADF and AIA (Trial 1) than medusahead. The high-energy supplements had concentrations of CP comparable to medusahead but lower concentrations of fiber. The silicaalfalfa diet had the greatest concentration of CP.

Trial 1-Medusahead in Late Vegetative/Early Reproductive Stage. Control lambs consumed more medusahead than supplemented lambs only during the first $2 \mathrm{~d}$ of testing (June 2 and 3; Group effect: $\mathrm{P}=0.27$; Group by Day; $\mathrm{P}=0.004$; Figure $1 \mathrm{~A}$ ). Medusahead intake for all groups was cyclic and declined relative to the initial $2 \mathrm{~d}$ of the trial (Day effect; $\mathrm{P}<0.0001$; Figure 1A).

No differences in supplement intake were measured between groups of lambs (Group effect: $\mathrm{P}=0.33$; Group by Day; $\mathrm{P}=0.53$; Figure $2 \mathrm{~A}$ ), but the Control group ate the greatest amounts of tall fescue hay $\left(22 \mathrm{~g} \mathrm{~kg}^{-0.75}\right.$; Group effect: $\mathrm{P}=0.10$; Group by Day; $\mathrm{P}=0.58$; Figure 2A). Supplemented lambs gained weight (greatest ADG values measured for Yeast supplementation), whereas control lambs lost weight (Group effect: $\mathrm{P}=0.07$; Figure 3A).

Choice Test-Medusahead vs. Tall Fescue Hay. When offered a choice between medusahead and tall fescue hay, lambs in Control, Ca Propionate, and Yeast consumed only 0.26, 0.44 , and $0.53 \mathrm{~g} \mathrm{~kg}^{-0.75}$ of medusahead (Group effect: $\left.\mathrm{P}=0.82 ; \mathrm{SEM}=0.3 \mathrm{~g} \mathrm{~kg}^{-0.75}\right)$, but they ingested 49, 45, and $46 \mathrm{~g} \mathrm{~kg}^{-0.75}$ of tall fescue hay (Group effect: $\mathrm{P}=0.72$; $\left.\mathrm{SEM}=4 \mathrm{~g} \mathrm{~kg}^{-0.75}\right)$, respectively.

Choice between Plant Parts. No differences between supplementation groups were measured regarding consumption of the plant parts offered during the choice test (Group effect; P $=0.70$; Group by Plant Part; $\mathrm{P}=0.60$ ). However, all lambs consumed (Plant Part effect; $\mathrm{P}=0.08$ )
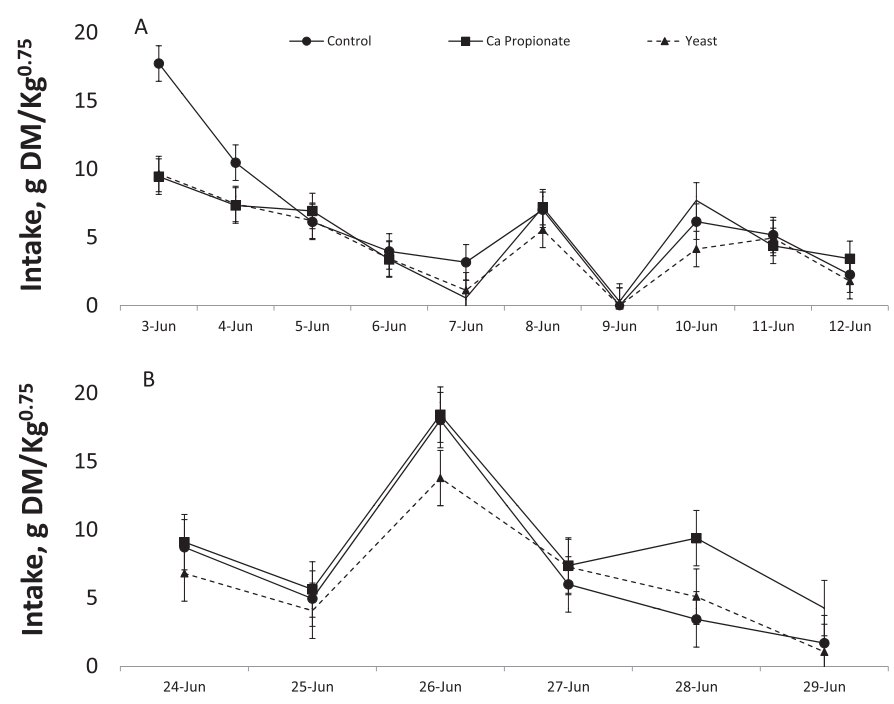

Figure 1. Intake of medusahead by three groups of lambs during two trials. Lambs were supplemented with: (1) Control (no supplement); (2) Ca Propionate (beet pulp:barley :Ca propionate, 67:30:3); or (3) Yeast (beet pulp:barley:yeast culture, $65: 30: 5)$. All animals then had ad libitum access to fresh-cut medusahead $\left(5 \mathrm{~h} \mathrm{~d}^{-1}\right)$ and subsequently a basal diet of tall fescue hay $\left(2 \mathrm{~h} \mathrm{~d}^{-1}\right)$ (Trial 1), or no basal diet (Trial 2). (a) Trial 1. Medusahead-late vegetative to mid-reproductive phenological stage. (b) Trial 2. Medusahead- late reproductive phenological stage. Values are means for 10 lambs; SE are represented by vertical bars.

and showed greater preference for the top parts (seedheads with awns) than for the mid parts (stems+leaves) (Plant Part effect; $\mathrm{P}<0.0001$; Figure 4).

Trial 2-Medusahead in Late Reproductive Stage. No differences between groups were measured regarding intake of medusahead (Group effect: $\mathrm{P}=0.36$; Group by Day; $\mathrm{P}=0.44$; Figures $1 \mathrm{~B}$ and $2 \mathrm{~B}$ ). Medusahead intake for all groups was cyclic and declined towards the end of the trial (Day effect; $\mathrm{P}<0.0001$; Figure 1B).

No differences in supplement intake were detected among groups of lambs, although lambs in Yeast tended to eat more supplement than lambs in $\mathrm{Ca}$ Propionate (Group effect: $\mathrm{P}=0.11$; Group by Day; $\mathrm{P}=0.11$; Figure 2B).

Control lambs lost $271 \mathrm{~g} \mathrm{~d}^{-1}$ whereas Yeast lambs lost $44 \mathrm{~g} \mathrm{~d}^{-1}$ during Trial 2 (i.e., when no basal diet of tall fescue hay was offered). In contrast, Ca Propionate lambs gained $49 \mathrm{~g} \mathrm{~d}^{-1}$ (Group effect: $\mathrm{P}<0.0001$; Figure 3B).

Choice Test-Medusahead vs. Tall Fescue Hay. When offered a choice between medusahead and tall fescue hay, lambs in Control, Ca Propionate, and Yeast consumed only 2.3, 1.8, and $2.1 \mathrm{~g} \mathrm{~kg}^{-0.75}$ of medusahead (Group effect: $\mathrm{P}=0.66$; SEM $\left.=0.4 \mathrm{~g} \mathrm{~kg}^{-0.75}\right)$, but they ingested 30,36 , and $27 \mathrm{~g}$ 

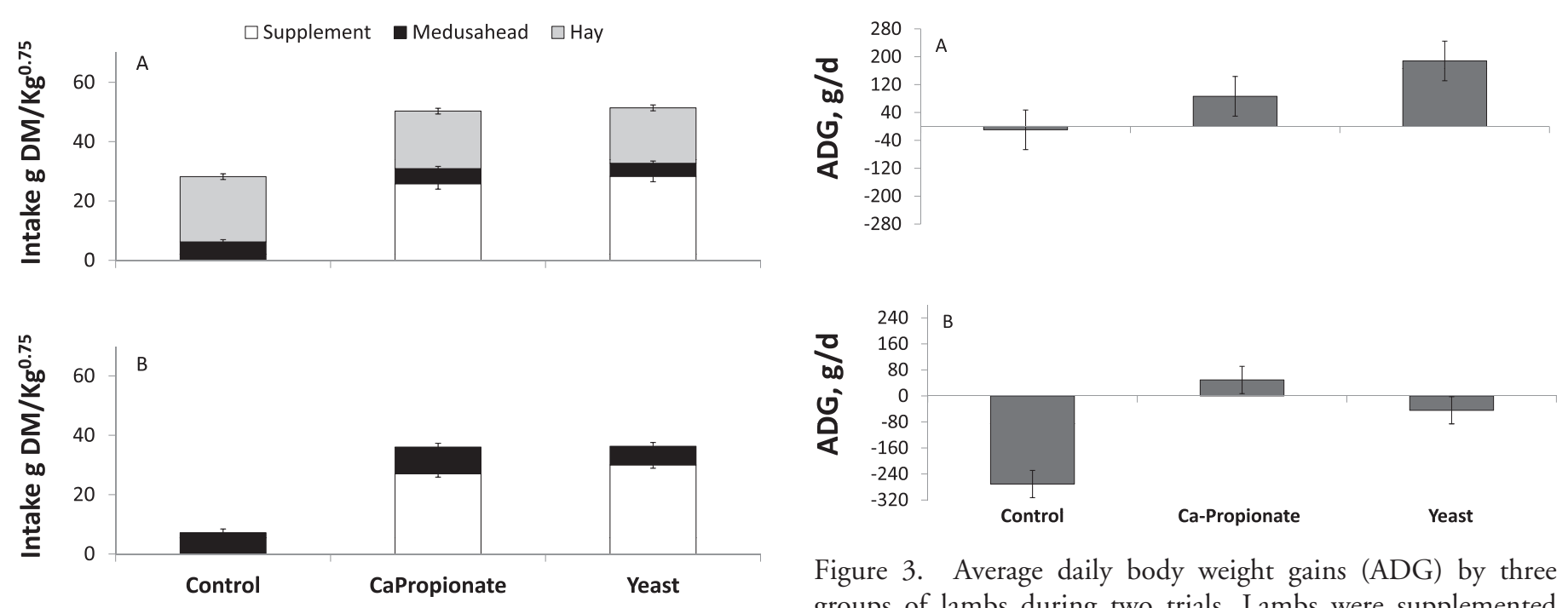

Figure 3. Average daily body weight gains (ADG) by three groups of lambs during two trials. Lambs were supplemented with: (1) Control (no supplement); (2) Ca Propionate (beet pulp : barley: Ca propionate, 67:30:3); or (3) Yeast (beet pulp : barley:yeast culture, $65: 30: 5)$. All animals then had ad libitum access to fresh-cut medusahead $\left(5 \mathrm{~h} \mathrm{~d}^{-1}\right)$ and subsequently a basal diet of tall fescue hay $\left(2 \mathrm{~h} \mathrm{~d}^{-1}\right)$ (Trial 1 ), or no basal diet (Trial 2). (a) Trial 1. Medusahead-late vegetative to mid-reproductive phenological stage. (b) Trial 2. Medusaheadlate reproductive phenological stage. Values are means for 10 lambs; SE are represented by vertical bars.

mid-reproductive phenological stage. (b) Trial 2. Medusaheadlate reproductive phenological stage. Values are means for 10 lambs; SE are represented by vertical bars.

$\mathrm{kg}^{-0.75}$ of tall fescue hay (Group effect: $\mathrm{P}=0.22$; $\mathrm{SEM}=$ $4 \mathrm{~g} \mathrm{~kg}^{-0.75}$ ), respectively.

Trial 3-Individual Variation and Intake of Silica. When lambs were separated onto two groups containing either Eaters or nonEaters, Eaters consistently ate more medusahead than the nonEaters group (Group effect; $\mathrm{P}<0.0001$; Figure 5A), with the typical cyclic pattern described before (Day and Group by Day effects; P $<0.0001$; Figure 5A). Intake of alfalfa hay (81 vs $77 \mathrm{~g} \mathrm{~kg}^{-0.75} ; \mathrm{SEM}=4$; Group effect; $\mathrm{P}=0.49$; Group by Day effect; $\mathrm{P}=0.26$ ) or tall fescue hay (14 vs $14 \mathrm{~g} \mathrm{~kg}^{-0.75}$; SEM $=2$; Group effect; $\mathrm{P}=0.88$; Group by Day effect; $\mathrm{P}=0.97$ ) did not differ between Eaters and nonEaters, respectively. However, when alfalfa intake was used as a covariate in the analysis with group and day in the model, a significant effect was observed of the covariate with group and lambs in the Eaters group ate more alfalfatsilica than lambs in the nonEaters group ( $\mathrm{P}=0.05$; Figure 5$)$.

\section{Discussion}

Effects of Supplements on Intake of and Preference for Medusahead. Prior research suggests that lambs
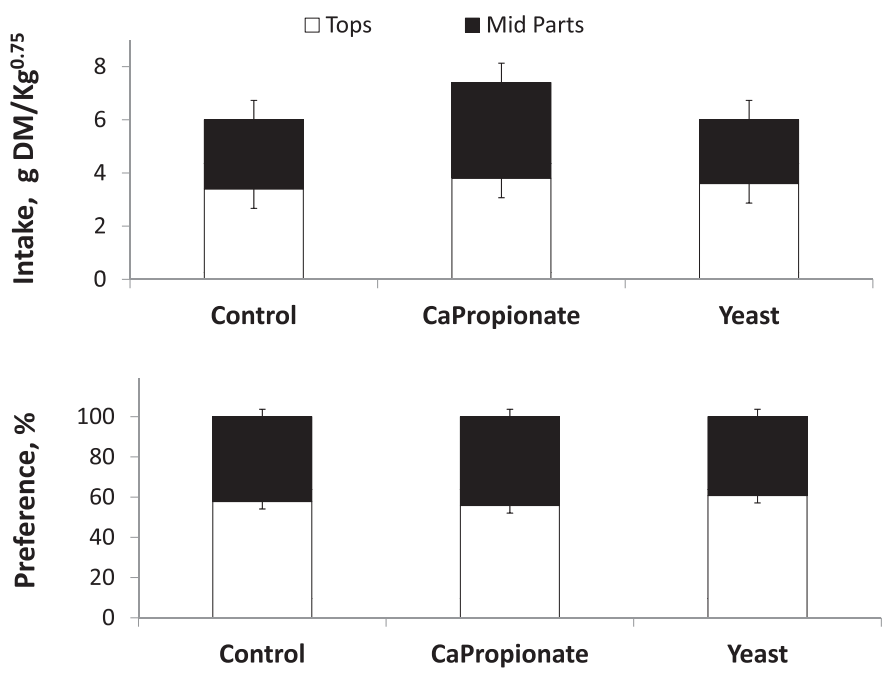

Figure 4. Intake and preference values by three groups of lambs offered a choice test between different medusahead plant parts: Tops (mostly seedheads and awns) vs. mid parts (mostly stems and leaves). Medusahead was in mid-reproductive phenological stage. For $10 \mathrm{~d}$ before the trial, lambs were supplemented with: (1) Control (no supplement); (2) Ca Propionate (beet pulp: barley: Ca propionate, 67:30:3); or (3) Yeast (beet pulp: barley: yeast culture, $65: 30: 5)$. Values are means for 10 lambs; $\mathrm{SE}$ are represented by vertical bars. 

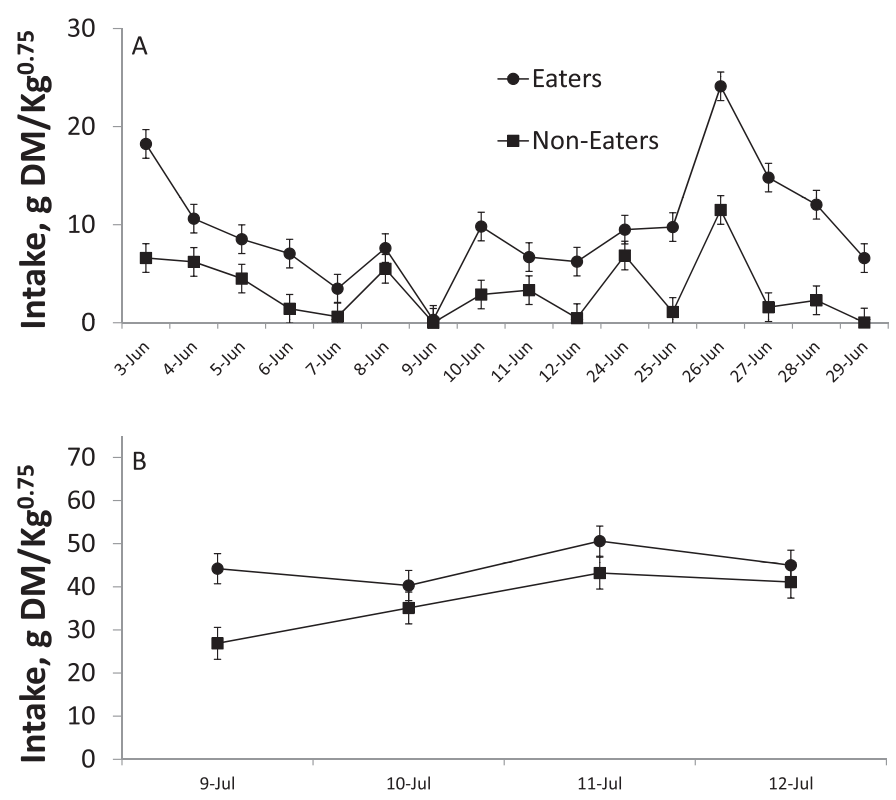

Figure 5. Intake of medusahead (a) and a silica-containing ration (alfalfa: silica, 97:3) (b) by two groups of lambs which showed high (Eaters) and low (nonEaters) intakes of medusahead during two prior trials. During these trials lambs were supplemented with: (1) Control (no supplement); (2) Ca Propionate (beet pulp:barley: Ca propionate, 67:30:3); or (3) Yeast (beet pulp : barley: yeast culture, 65:30:5). All animals then had ad libitum access to fresh-cut medusahead $\left(5 \mathrm{~h} \mathrm{~d}^{-1}\right)$ and subsequently a basal diet of tall fescue hay $\left(2 \mathrm{~h} \mathrm{~d}^{-1}\right)$ (Trial 1), or no basal diet (Trial 2). Values are means for 10 lambs; SE are represented by vertical bars.

receiving energy-rich supplements tend to eat more medusahead than lambs supplemented with protein-rich supplements or unsupplemented animals (Hamilton et al. 2015). Thus, energy-rich supplements were used in the study with the addition of $\mathrm{Ca}$ propionate or yeast culture as potential treatments to further enhance utilization of medusahead by sheep as well animal performance. Ruminants ingesting lowquality forages like medusahead may benefit from supplements containing glucogenic precursors derived from rumen fermentation like propionate. For instance, supplements containing $\mathrm{Ca}$ propionate enhance pregnancy rates and decrease days to resumption of estrous in cows grazing lowquality pastures (Mulliniks et al. 2011). The addition of yeast (e.g., Saccharomyces cerevisiae fermentation products) to the rumen environment increases cellulose degradation (Williams et al. 1991) by stimulating growth of fiberdigesting rumen microorganisms (Callaway and Martin 1997; Mao et al. 2013) and increasing microbial protein flow to the duodenum (Hristov et al. 2010). Consistent with this, yeast cultures increase intake, NDF and CP degradation in steers grazing rangelands (Olson et al. 1994). Nevertheless, supplementation during Trials 1 and 2 did not lead to increases in intake typically observed when a poorquality feed is supplemented with a high-quality feed (Figure 2). Positive associative effects are observed when low-quality forages are supplemented with protein, which allows for better utilization of those forages. Negative associative effects often occur when high-grain diets are diluted by forages, which leads to reductions in DM digestibility (Van Soest 1994). In the present study, intake of medusahead was fairly constant across groups of lambs regardless of the availability and type of supplement. Only simple additive effects on total food intake were apparent when lambs were offered supplement+medusahead relative to when lambs were only offered medusahead (Control). Likewise, tall fescue hay intake was fairly constant across groups of lambs, although Control lambs ate slightly greater amounts of hay likely because they received no supplementation.

While Ca-Propionate and Yeast supplementation did not modify intake of medusahead relative to the Control treatment, ADG for supplemented lambs were always greater than for nonsupplemented animals. Thus, supplementing animals with Ca propionate or yeast in targeted grazing treatments has the potential to achieve similar levels of medusahead intake and better levels of production than forcing animals to ingest the weed in fenced areas for short periods of time without supplementation. Producers are more likely to use mature breeding livestock for targeting grazing treatments given the resulting sacrificed body weight gains from growing animals forced to consume unpalatable weeds as reported in this study for Control animals. However, our results suggest that supplementation can overcome such constraint and potentially improve the nutrition and welfare of grazing animals without depressing the ingestion of medusahead.

Preference tests support the notion that ruminants strongly avoid medusahead, particularly during the early to late reproductive stages of the plant (Bovey et al. 1961; Murphy and Turner 1959). Supplements did not modify preference for the weed, as levels of avoidance were comparable across treatments with (Trial 1) or without (Trial 2) a basal diet of tall fescue hay. Nevertheless, the notion that awns are a cause for weed avoidance as the plant becomes too prickly was not supported in the present study. On the contrary, lambs preferred the top parts of the plant (mostly seedheads with awns) to the rest of the plant, likely due to the greater content of $\mathrm{CP}$ and lower concentrations of ADF and AIA in the former than in the mid part of the plant (Table 1).

Consistent with previous research (Hamilton et al. 2014), intake of medusahead was low and cyclic across days. Cyclic patterns of food intake are characteristic of toxin-containing plants, as peaks of food intake are followed by days of restricted food consumption (nadirs) required by animals to detoxify the levels of secondary 
compounds ingested in previous meals (Foley et al. 1999; Pfister et al. 1997). Nevertheless, intraruminal infusions of medusahead in a previous study did not condition a food aversion in sheep which suggests toxins are not a reason for the low levels of intake displayed by ruminants offered medusahead (Hamilton et al. 2014). Alternatively, the cyclical nature of medusahead intake suggests a constraint induced by a filling effect due to fiber content or fiber characteristics. Voluntary dry matter intake of forages by ruminants is limited by rumen distention, which is the result of restricted flow of digesta through the gastrointestinal tract (Allen 1996). Since NDF typically ferments and passes through the digestive tract at a slower rate than other nonfibrous feed constituents, this fraction has been regarded as the best single chemical predictor of intake (Allen 1996). The NDF content of medusahead was comparable to that of tall fescue hay. Yet, lambs ingested considerably greater amounts of tall fescue hay than medusahead during Trial 1 , even during a much shorter period of time ( 2 vs. $5 \mathrm{~h} \mathrm{~d}^{-1}$ ) and they clearly preferred tall fescue hay to medusahead during preference tests of Trials 1 and 2 . In addition to fiber content, the fiber filling effect in the rumen varies with other factors such as particle fragility (McLeod and Minson 1988), and rate and extent of NDF digestion (Allen 1996). Low particle fragility and a reduced rate of digestion of NDF in medusahead relative to other grasses and the concomitant increase in rumen fill may contribute to explain the low and cyclic pattern of medusahead intake in ruminants.

High content of silica in plant tissues has been proposed as a defense mechanism against herbivory (McNaughton et al. 1985) and concentrations of silica in medusahead are high, as suggested by the high concentrations of acid insoluble ash (AIA) found in the present study for medusahead (Table 1). Nevertheless, animals had a greater intake capacity for tall fescue hay-like in the present studyand other forages that contain high silica concentrations (e.g., rice straw; Van Soest 2006) than for medusahead. Finally, daily changes in the nutritional composition of the harvested plant material may have introduced another source of variation which contributed to the cyclic patterns of medusahead intake reported in this study.

Individual Variation, Silica and Ingestion of Medusahead. Differences in food intake depend in part on physiological and morphological variations among individuals (reviewed by Provenza et al. 2003). Marked differences are common even among uniform groups of ruminants regarding requirements for nutrients (Scott and Provenza 1999) and abilities to cope with toxins (Provenza et al. 1999). Consistent with this notion, clear and consistent differences occurred in this study regarding the lambs' ability to ingest medusahead. Such differences have been recorded in prior studies for medusahead (e.g.,
Hamilton et al. 2014; Montes and Villalba, unpublished results) and other forages (e.g., Doran et al. 2007), which suggest physiological/morphological differences among individuals are a significant variable affecting medusahead intake. In fact, differences among individuals were more consequential at influencing medusahead intake than any of the supplementation treatments assayed. Future research should focus on understanding these differences in order to achieve greater levels of medusahead use during targeted grazing treatments. One potential variable, which may influence a greater utilization of the weed may be related to the animals' ability to ingest silica, as lambs with high intake of medusahead (Eaters) also consumed greater amounts of a silica-containing diet than lambs with low intake of the weed (nonEaters) (Figure 5). Future research should also be conducted in range settings to determine if the patterns of intake and individual variation found in these controlled feed trials transfer to larger spatial scales.

\section{Acknowledgments}

Research was funded by the National Institute of Food and Agriculture (NIFA) USDA (Award 2009-35101-05360). This paper is published with the approval of the Director, Utah Agricultural Experiment Station, and Utah State University, as journal paper number 8738 . We acknowledge R. Stott for veterinary services and S. Hunt and T. Hamilton for technical support.

\section{Literature Cited}

Allen MS (1996) Physical constraints on voluntary intake of forages by ruminants. J Anim Sci 74:3063-3075

AOAC (2000) Official Methods of Analysis. 17th ed. Gaithersburg, MD: Assoc. Offic. Anal. Chem. 2200 p

Bovey RW, LeTourneau D, Erickson LC (1961) The chemical composition of medusahead and downy brome. Weeds 9:307-311

Callaway ES, Martin SA (1997) Effects of a Saccharomyces cerevisiae culture on ruminal bacteria that utilize lactate and digest cellulose. J Dairy Sci 80:2035-2044

Davies KW, Johnson DD (2008) Managing medusahead in the intermountain west is at a critical threshold. Rangelands 30:13-15

Davy J, Laca EA, Forero L (2009) Medusahead: What is being tested to reduce it? Northern California Ranch Update, Vol. 3. University of California Cooperative Extension. $4 \mathrm{p}$

Doran MP, Laca EA, Sainz RD (2007) Total tract and rumen digestibility of mulberry foliage (Morus alba), alfalfa hay and oat hay in sheep. Anim Feed Sci Technol 138:239-253

Doran MP (2008) Luring sheep with molasses. Western SARE Professional Development Program Report. www.sare.org/content/down load/1627/11466/FW06_304_txt.pdf. Accessed January 26, 2014

Foley WJ, Iason GR, McArthur C (1999) Role of plant secondary metabolites in the nutritional ecology of mammalian herbivores: how far have we come in 25 years? Pages 130-209 in Proceedings of the Fifth International Symposium on the Nutrition of Herbivores, Nutritional Ecology of Herbivores. Savory, IL: American Society of Animal Science 
Hamilton T, Burritt EA, Villalba JJ (2015) Assessing the impact of supplements, food aversions, and silica on medusahead (Taeniatherum caput-medusae (L.) Nevski) use by sheep. Small Rum. Res. 124:45-54

Hristov AN, Varga G, Cassidy T, Long M, Heyler K, Kaenati SKR, Corl B, Hovde J, Yoon I (2010) Effect of Saccharomyces cerevisiae fermentation product on ruminal fermentation and nutrient utilization in dairy cows. J Dairy Sci 93:682-692

Mao HL, Wang JK, Liu JX, Yoon I (2013) Effects of Saccharomyces cerevisiae fermentation product on in vitro fermentation and microbial communities of low-quality forages and mixed diets. J Anim Sci 91:3291-3298

McLeod MN, Minson D (1988) Large particle breakdown by cattle eating ryegrass and alfalfa. J Anim Sci 66:992-999

McNaughton SJ, Tarrants JL, McNaughton MM, Davis RH (1985) Silica as a defense against herbivore and a growth promoter in African grasses. Ecology 66:528-535

Mulliniks JT, Cox SH, Kemp ME, Endecott RL, Waterman RC, VanLeeuwen DM, Petersen MK (2011) Protein and glucogenic precursor supplementation: A nutritional strategy to increase reproductive and economic output. J Anim Sci 89:3334-3343

Murphy AH, Turner D (1959) A study on the germination of medusahead seed. Pages 6-10 in The Bulletin 48. California State Department of Agriculture

Olson KC, Caton JS, Kirby DR, Norton PL (1994) Influence of yeast culture supplementation and advancing season on steers grazing mixed-grass prairie in the northern Great Plains: II. Ruminal fermentation, site of digestion, and microbial efficiency. J Anim Sci $72: 2158-2170$

Pfister JA, Provenza FD, Manners GD, Gardner DR, Ralphs MH (1997) Tall larkspur ingestion: can cattle regulate intake below toxic levels? J Chem Ecol 23:759-777
Provenza FD, Villalba JJ, Augner M (1999) The physics of foraging. III. Pages 99-107 in Proceedings of the XVIII International Grassland Congress, Saskatchewan Agriculture and Food, Saskatoon, SK

Provenza FD, Villalba JJ, Dziba LE, Atwood SB, Banner RE (2003) Linking herbivore experience, varied diets, and plant biochemical diversity. Small Rum Res 49:257-274

Scott LL, Provenza FD (1999) Variation in food selection among lambs: effects of basal diet and foods offered in a meal. J Anim Sci 77: 2391-2397

Swenson CF, LeTourneau D, Erickson LC (1964) Silica in medusahead. Weeds 12:16-18

Van Keulen J, Young BA (1977) Evaluation of acid-insoluble ash as a natural marker in ruminant digestibility studies. J Anim Sci 44: 282-287

Van Soest PJ, Robertson JB, Lewis BA (1991) Methods for dietary fiber, neutral detergent fiber, and nonstarch polysaccharides in relation to animal nutrition. J Dairy Sci 74:3583-3597

Van Soest PJ (1994) Nutritional Ecology of the Ruminant. 2nd ed. Ithaca, NY: Cornell University Press. $476 \mathrm{p}$

Van Soest PJ (2006) Rice straw, the role of silica and treatments to improve quality. Anim Feed Sci Technol 130:137-171

Williams PEV, Tait CAG, Innes GM, Newbold CJ (1991) Effects of the inclusion of yeast cultures (Saccharomyces cereuisiae plus growth medium) in the diet of dairy cows on milk yield and forage degradation and fermentation patterns in the rumen of steers. J Anim Sci 69:3016-3026

Zimmerman J, Johnson WS, Eiswerth ME (2002) Medusahead: Economic Impact and Control in Nevada. University of Nevada Extension Service. Reno, Nevada. Fact Sheet FS-02-37. 4 p

Received October 18, 2014 and approved January 30, 2015. 